\title{
La question de la sécession dans une fédération: Une critique des mauvaises interprétations
}

\author{
Åsbjørn Melkevik
}

\section{Introduction : la question de la sécession}

Nous avons intitulé notre essai «la question de la sécession» à dessein; cela implique nécessairement qu'il y ait une question à poser et sur laquelle il convient de réfléchir. Michael Ignatieff, mesurant bien toute l'importance de la question de la sécession au Canada, écrivait : «Members of my generation have spent their entire adult political life wondering whether the country either can or deserves to survive. ${ }^{1} \gg$ En effet, cette question suscite des passions profondes, qui malheureusement entraînent souvent, de la part du sujet passionné, soit un appui total ou un rejet total d'une possible sécession. Au contraire, il ne faut pas rejeter a priori l'interrogation ou affirmer simplement qu'elle est déjà réglée. Autant les attitudes rejetant complètement la possibilité d'une sécession que les attitudes attribuant à la sécession une certaine valeur transcendantale inévitable sont dangereuses pour le fédéralisme et pour la discussion démocratique devant nécessairement précéder un retrait formel d'une entité fédérée. Ainsi, le principal danger pour le fédéralisme n'est pas tant la possibilité d'une sécession en soi, que la radicalisation des idées sur la sécession. D'un côté, il est dangereux de tomber dans le piège du holisme, soit la pensée voulant que le tout soit plus grand que la somme de ses parties; d'où l'idée erronée voulant que les provinces n'ont d'importance que par rapport

\footnotetext{
${ }^{1}$ Michael IGNATIEFF, Blood and Belonging. Journeys Into The New Nationalism, Toronto, Penguin Canada, 1993, page 174.
}

ÅSBJØRN MELKEVIK est présentement étudiant au baccalauréat en science politique à l'Université Laval d'où il graduera en mai 2011. Il a l'intention de poursuivre des études supérieures. Ses intérêts portent sur la philosophie politique ainsi que l'histoire des idées et plus particulièrement sur les questions de parlementarisme et de fédéralisme. 


\section{Åsbjørn Melkevik}

La question de la sécession dans une fédération: Une critique des mauvaises interprétations

à l'État central. D'un autre côté, l'idée que les parties sont inlassablement opprimées par le tout et que leurs réelles valeurs ou identités ne peut exister ou survivre que par une éventuelle séparation du tout est très certainement erronée, dans la plupart des cas. Ces deux types de préconception entraînent toutes sortes d'arguments idéologiques inappropriés qui tentent de fixer ou de «régler » la question de la sécession une fois pour toute ; au contraire, selon nous, la question doit rester ouverte.

Dans le présent essai, nous nous attarderons donc à réfuter les mauvaises interprétations de la question de la sécession voulant détruire cette possibilité d'une intelligence politique - d'une raison prudentielle aristotélicienne. Nous tenterons, dans notre première partie, de souligner l'insuffisance du Droit dans le discours concernant les sécessions. Dans notre deuxième partie, nous envisagerons, à la suite du Renvoi de la Cour suprême du Canada, la sécession comme une procédure devant impliquer un dialogue - nous verrons comment cette notion de négociation fut dévaluée autant par une loi fédérale qu'une loi provinciale québécoise. Dans notre troisième partie, nous critiquerons les théories s'attachant à mettre trop l'accent sur le côté négatif des projets sécessionnistes, soit principalement la théorie de Stéphane Dion de la peur et de la confiance ; nous terminerons par proposer notre vision positive.

\section{L'insuffisance du Droit dans le discours sur la sécession}

L'évocation de la notion de « Droit» est un argument qui revient sans cesse dans les discussions entourant la possibilité d'une sécession pour une entité fédérée. Or la notion de Droit - peu importe ce que l'on entend par là, c'est-à-dire soit le «droit international ", soit le droit étatique - ne saurait véritablement être concluante concernant la question et risque bien de se réduire à être une simple «justification » d'une position idéologique. Plutôt que le concept de Droit, c'est la question de la procédure politique qui doit nous préoccuper - et dont nous reparlerons dans notre prochaine section. Or, le fait que le droit et les règles procédurales peuvent être créés par le même organe étatique - la Cour suprême, dans le cas du Canada - ne doit en aucun cas 


\section{Åsbjørn Melkevik La question de la sécession dans une fédération: Une critique des mauvaises interprétations}

nous faire mélanger la question procédurale avec la question du Droit ${ }^{2}$. Dans la présente section, nous nous attarderons donc à montrer que le recours à la notion de Droit international n'est souvent simplement pas approprié.

\section{Le droit à l'autodétermination des nations}

Le droit international inclut un "droit à l'autodétermination des nations », ou encore un " droit des peuples à disposer d'eux-mêmes », qui - selon certains intellectuels - justifierait un «droit» à la sécession. Les justifications invoquées de ce principe ne nous intéressent pas ici en tant que telles, sinon l'idée qu'à un «peuple reconnu ${ }^{3}$ » correspondent des droits. Or, cette conception nous semble largement erronée. Naturellement la reconnaissance de l'existence d'une «nation » ne fournit aucun « droit » en soi. Une nation est simplement un fait politique avec lequel il convient de composer ; le fédéralisme ou la décentralisation sont ainsi des outils qui permettront d'assurer que la nation en question puisse effectivement participer à l'exercice du pouvoir. S'il y a une indépendance, elle se fera par des citoyens et non par une immanence ou par une transcendance découlant d'un fait national distinct à l'intérieur d'une fédération. Le fait d'être une nation distincte ne justifie donc aucunement une indépendance ${ }^{4}$. En ce sens, nous pourrions dire que la justification d'un «droit de sécession» pour les nations se situe dans ce que Hume appelait le paralogisme naturaliste. Il y a passage non logique de la factualité à la normativité ; du fait national au projet de sécession. Les faits ne peuvent pas justifier des intentions, ce serait inscrire dans la réalité des intentions qui n'y sont pas. $\mathrm{Au}$ contraire, les sécessions doivent se comprendre comme des projets collectifs -

\footnotetext{
${ }^{2}$ Le Droit ne doit pas devenir un terme générique, qui perd ainsi son sens propre; par exemple, pour Kelsen toute production de l'État est soit création ou application du droit. Cela a pour conséquence que le droit devient un mot valise, sans utilité pour faire des distinctions conceptuelles. Hans KELSEN, Théorie générale du droit et de l'État, Paris, Bruylant, Librairie Générale de Droit et de Jurisprudence, E.J.A., 1997, page 318.

${ }^{3}$ Mentionnons ici que l'idée de reconnaissance est aussi problématique. Cela rentre, dans une certaine mesure, en contradiction avec l'idéal libéral de l'État qui ne reconnaît pas de groupe particulier, mais s'efforce de maintenir une séparation avec la société civile.

${ }^{4}$ «It is important to reject the notions that every ethnically or culturally distinct people, nation, or group has an automatic right to its own state or that ethnically homo generous states are inherently desirable. » Hurst HANNUM, «The Specter of Secession: Responding to Claims for Ethnic Self- Determination », Foreign Affairs, vol. 77, no 2, mars - avril, 1998, pp. 16.
} 


\section{Åsbjørn Melkevik La question de la sécession dans une fédération: Une critique des mauvaises interprétations}

comme nous le défendrons dans notre troisième section - ce qui implique un certain jugement des citoyens; ce jugement ne peut pas être remplacé par un droit.

\section{Les nations menacées de destruction}

Être une nation ne donne ainsi pas de «droits » de sortir de l'État de droit, de manière purement artificielle. Cependant, certains diront que ce droit de sécession peut être reconnu lorsqu'une entité fédérée est menacée de destruction par l'État central ; la primauté de la procédure politique ou constitutionnelle peut alors être écartée sans trop de risque. Concernant le droit à l'autodétermination des nations, Michael Ignatieff, qui reconnaît ce droit, écrit : "Mais l'autodétermination n'implique pas nécessairement le droit à la sécession. La sécession et l'indépendance se justifient pour une nation menacée de destruction, et seulement si la possession des pouvoirs d'un États souverain garantit son salut. ${ }^{5} \gg$ Il convient alors de se poser une question : le fait qu'une nation soit menacée de destruction donne-t-il un « droit » à la sécession ? Selon nous, la réponse est non. Il ne peut pas y avoir une telle chose qu'un «droit» de se protéger. À ce sujet, Hobbes soulignait déjà que lorsque l'on est menacé de mort par quelqu'un - y compris par l'État - on sort automatiquement de l'État de droit et peut naturellement défendre sa vie ${ }^{6}$. Bien que les parallèles entre les individus et les groupes soient souvent trompeurs, nous croyons qu'ici la comparaison est juste. Si une province ou une nation est menacée par l'État fédéral - dans le sens physique ou politique du terme et non pas culturellement -, la notion de droit n'est simplement pas nécessaire ${ }^{7}$ : la sécession sera alors un moyen tout naturel, voire politique, parmi d'autres de se protéger.

En conséquence, l'argument du droit de sécession, lorsqu'il se réfère à un droit international, n'est souvent simplement pas approprié ; soit le droit de sécession n'est pas vraiment utile, comme dans le cas des nations menacées physiquement, soit il repose

\footnotetext{
${ }^{5}$ Michael IGNATIEFF, La Révolution des droits, Montréal, Boréal, 2001, page 121.

${ }^{6}$ Thomas HOBBES, Léviathan, Paris, Éditions Sirey, 1971, page 128. «il est interdit aux gens de faire ce qui mène à la destruction de leur vie ou leur enlève le moyen de la préserver, et d'omettre ce par quoi ils pensent qu'ils peuvent être le mieux préservés. »

${ }^{7}$ Dans certains cas, la notion pourrait être utile ; les nombreux démocides du XXe siècle en témoignent.
} 


\section{Åsbjørn Melkevik}

\section{La question de la sécession dans une fédération: Une critique des mauvaises interprétations}

sur une interprétation trop large du droit à l'autodétermination ${ }^{8}$ et n'est que du wishful thinking : « Despite continued claims to a "right" of secession by groups in Asia, Africa, Europe, and the former Soviet Union, no such right has yet been recognized by the international community. International law does not prohibit secession, whether voluntary or violent, but it has neither recognized a right to secede nor identified even tentatively the conditions that might give rise to such a right in the future. ${ }^{9}$ " Ainsi, dans l'éventualité d'une sécession à l'intérieur d'une fédération, il ne faut pas se référer à un droit international, mais bien prioritairement à la procédure démocratique interne.

\section{La question procédurale d'une sécession}

La sécession dans un régime fédéral est, selon la définition de John R. Wood ${ }^{10}$, un retrait formel d'un membre par rapport à l'autorité centrale. Cette définition a l'avantage de ne pas faire référence à de grands principes et de rester somme toute assez simple. Or, le mot « formel » doit être souligné ; la formalité implique nécessairement une procédure de dévaluation du pouvoir vers la nouvelle entité politique. Si nous avons rejeté le droit international comme étant non pertinent dans l'éventualité d'une sécession, l'importance de la procédure doit être soulignée et ne surtout pas être confondue avec la notion de droit. Un droit implique quelque chose qui est dû et qui peut être revendiqué à une autorité - la sécession n'est due à personne et ne peut pas être revendiquée à une autorité quelconque. Par ailleurs, une procédure implique simplement que lorsque l'on veut atteindre un certain but, il est nécessaire de suivre une démarche formelle pour atteindre ce but. Il peut être avantageux de comprendre le politique comme essentiellement procédural; ainsi les différentes variations de systèmes politiques sont autant de procédures pour atteindre certains buts : la démocratie, le fédéralisme, le parlementarisme et même le totalitarisme peuvent être compris dans cet esprit. La sécession en tant

\footnotetext{
${ }^{8}$ Il ne faut pas confondre les politiques adoptées par l'ONU, en vue de favoriser le processus de décolonisation, avec un droit reconnu et invocable par tous ; cela n'est pas un droit de sécession invocable. Il semble assez clair que le Québec n'est pas une colonie et ne peut donc pas bénéficier des politiques anticolonialistes.

${ }^{9}$ Hurst HANNUM, op. cit., page 13-14.

${ }^{10}$ WOOD, John R., « Secession: A Comparative Analytical Framework », Canadian Journal of Political Science, vol. 16, no 1, 1981, page 110.
} 
qu'événement politique peut par conséquent aussi être envisagée en termes de procédures. Nous allons, dans la présente section, premièrement nous expliquer sur ce point de politique procédurale, pour deuxièmement examiner la manière dont cette procédure se déploie au Canada et comment elle fut interprétée par la Cour suprême et dévaluée par les réactions fédérale et provinciale.

\section{Le fédéralisme procédural}

Selon le philosophe Karl Popper : «Il est faux [...] de mettre l'accent [...] sur la question: "Qui doit gouverner? Le peuple (la plèbe) ou les quelques meilleurs? Les (bons) travailleurs ou les (mauvais) capitalistes ? La majorité ou la minorité ? La gauche, la droite, ou le parti du centre?" Toutes ces questions sont mal posées. Car il importe peu de savoir qui gouverne, tant que l'on peut se défaire du gouvernement sans effusion de sang. ${ }^{11} »$ Le problème principal de la démocratie serait donc : «comment doit-on gouverner », soit un raisonnement insistant sur les limites de l'exercice du pouvoir et sur le processus pacifique du changement de gouvernement. Il y a en effet une importante dichotomie entre un questionnement qui insiste sur une fin - la prise du pouvoir par le prolétariat par exemple - soit un raisonnement foncièrement téléologique, et un questionnement insistant sur les moyens, soit un raisonnement procédural. Le fédéralisme doit être compris dans le même ordre d'idée que la démocratie, c'est-à-dire comme essentiellement procédural. Ce système offre une autre réponse à la question de «comment doit-on gouverner», qui n'est évidemment pas incompatible avec la démocratie - bien au contraire -, mais qui se démarque d'un modèle d'union centralisée, qui serait une autre réponse appropriée à la question politique ${ }^{12}$. Ainsi, le fédéralisme est une procédure visant à assurer la fonctionnalité d'un pays confronté par exemple à un immense territoire; à une pluralité de nations ou d'identités ${ }^{13}$; à des problèmes de sécurité ; ou à des problèmes économiques de marché intérieur etc. Dans le cadre d'une

\footnotetext{
${ }^{11}$ Karl POPPER, Toute vie est résolution de problèmes, tome 2, Arles, Actes Sud, 1998, pages 74-75.

${ }^{12}$ Carl J. FRIEDRICH, Trends of Federalism in Theory and Practice, New York; Washington; Londres, Praeger Publishers, 1968, pages 3-4.

${ }^{13}$ « Federalism thus provides the only voluntary approach to the task of coordinating disparate national elements. » Ibid., page 34 .
} 


\section{Åsbjørn Melkevik La question de la sécession dans une fédération: Une critique des mauvaises interprétations}

fédération, la sécession implique par conséquent qu'une entité fédérée envisage une réponse contradictoire ${ }^{14}$, à celle donnée par la fédération, concernant la question politique du vivre ensemble - cela implique un projet de société. La sécession, comme nous le défendrons, doit être d'une part projet et d'autre part procédure. La procédure d'une sécession doit, comme nous allons le voir avec le Renvoi de la Cour suprême du Canada, faire référence essentiellement à la notion de négociation; il doit y avoir un dialogue entre les acteurs d'une fédération, puisqu'il s'agit ici d'une question aussi essentielle qu'un changement de citoyenneté.

\section{Le Renvoi de la Cour suprême sur la sécession du Québec}

Il est intéressant, pour examiner l'importance de la procédure qui doit précéder une sécession, de se pencher sur le Renvoi de la Cour suprême sur la sécession du Québec. La première question posée à la Cour suprême - celle qui est intéressante pour notre propos - porte sur la possibilité de l'unilatéralité d'une sécession: «1. L'Assemblée nationale, la législature, ou le gouvernement du Québec peut-il, en vertu de la Constitution du Canada, procéder unilatéralement à la sécession du Québec du Canada $?^{15} »$.

La Cour suprême répond, de manière assez sage, en rappelant un fait essentiel, mais souvent oublié : «La démocratie, toutefois, signifie davantage que la simple règle de la majorité. ${ }^{16}$ »Elle ne rejette évidemment pas cette règle, mais souligne que la majorité n'est pas suffisante - on ne peut pas sortir de l'État de droit par une simple majorite $^{17}$ : «Le Québec ne pourrait, malgré un résultat référendaire clair, invoquer un

\footnotetext{
${ }^{14}$ «Quand les objectifs locaux particularistes sont suffisamment forts et cohérents pour maintenir ou pour constituer en groupes autonomes les subdivisions territoriales du pays, alors la structure politique adéquate est la Confédération. En revanche, l'Organisation fédérale apparaît quand les objectifs opposés (intérêts, traditions, intentions) ne sont pas encore, ou ne sont plus suffisamment forts pour maintenir des éléments autonomes. » Carl J. FRIEDRICH, La démocratie constitutionnelle, Paris, Presses Universitaires de France, 1958, page 164.

${ }^{15}$ Renvoi relatif à la sécession du Québec, [1998] 2 R.C.S. 217.

${ }^{16}$ Ibid.

${ }^{17} \mathrm{Au}$ contraire de Carl Schmitt, dont Renaud Baumert synthétise ainsi la pensée : «La pensée démocratique suppose - principe d'identité oblige - que le peuple soit souverain et qu'aucun frein ni
} 


\section{Åsbjørn Melkevik La question de la sécession dans une fédération: Une critique des mauvaises interprétations}

droit à l'autodétermination pour dicter aux autres parties à la fédération les conditions d'un projet de sécession. Le vote démocratique, quelle que soit l'ampleur de la majorité, n'aurait en soi aucun effet juridique et ne pourrait écarter les principes du fédéralisme et de la primauté du droit, les droits de la personne et des minorités, non plus que le fonctionnement de la démocratie dans les autres provinces ou dans l'ensemble du Canada. ${ }^{18}$ » La Cour suprême fait ensuite quelque chose de remarquable politiquement en insistant sur la notion de négociation. C'est le politique qui doit prendre ses responsabilités; les différents acteurs de la fédération doivent, dans l'éventualité d'une majorité claire donnant une légitimité à un projet sécessionniste clair, engager un dialogue sur les conditions d'une sécession. "Les négociations qui suivraient un tel vote porteraient sur l'acte potentiel de sécession et sur ses conditions éventuelles si elle devait effectivement être réalisée. Il n'y aurait aucune conclusion prédéterminée en droit sur quelque aspect que ce soit. ${ }^{19}$ " La phrase précédente exprime magnifiquement que ce n'est donc pas le droit, mais la politique qui doit s'occuper de la question d'une sécession. La principale procédure que nous pouvons faire ressortir de ce Renvoi est qu'une sécession doit nécessairement impliquer un dialogue sincère et sérieux. Cela permet ainsi d'introduire ce que l'on pourrait appeler, à la suite de Jürgen Habermas, la démocratie délibérative, ou dans ce cas-ci le fédéralisme délibératif.

Le Renvoi de la Cour suprême était, selon nous, essentiellement intelligent et réfléchi ${ }^{20}$ dans son utilisation de la notion de négociation. Or, le portrait se gâte par la suite avec les deux réactions à ce Renvoi, respectivement fédérale et provinciale, soit la Loi donnant effet à l'exigence de clarté formulée par la Cour suprême du Canada dans son avis sur le Renvoi relatif à la sécession du Québec, ci-après nommée Loi sur la clarté,

contrepoids ne puisse entraver son exercice du pouvoir. Par définition même : le libéralisme est modération, la démocratie est radicalisme. » Renaud BAUMERT, « Carl Schmitt contre le parlementarisme weimarien. Quatorze ans de rhétorique réactionnaire », Revue française de science politique, volume 58, no 1, 2008, pages 11-12.

${ }^{18}$ Renvoi relatif à la sécession du Québec, [1998] 2 R.C.S. 217.

${ }^{19}$ Ibid., Nous soulignons.

${ }^{20}$ Mentionnons par ailleurs que l'identification de la nécessité de modifier la Constitution en cas de sécession est quelque peu ridicule, lorsque l'on sait la complexité d'une telle tâche au Canada. La loi sur la clarté résume ce principe ainsi : « au Canada, la sécession d'une province, pour être légale, requerrait une modification à la Constitution du Canada ». 


\section{Åsbjørn Melkevik}

\section{La question de la sécession dans une fédération: Une critique des mauvaises interprétations}

et la Loi sur l'exercice des droits fondamentaux et des prérogatives du peuple québécois et de l'État du Québec. Le problème avec la Loi sur la clarté de Stéphane Dion est justement qu'elle met l'accent sur la clarté, quand l'aspect le plus important du Renvoi est, selon nous, la notion de négociation. L'accent mis sur la clarté n'est pas innocent ; c'est évidemment à l'avantage du gouvernement central de pouvoir décider si une question est claire et si une majorité est claire. La Chambre des communes se réserve la possibilité d'examiner : «a) l'importance de la majorité des voix validement exprimées en faveur de la proposition de sécession; b) le pourcentage des électeurs admissibles ayant voté au référendum; c) tout autres facteurs ou circonstances qu'elle estime pertinents ${ }^{21} »$. Voilà qui est tout de même assez vague et pas très clair pour une loi sur la clarté ; le message est que le parti sécessionniste doit être clair, mais que le gouvernement fédéral n'est pas obligé de l'être quant à ses critères évaluatifs. Cela permet au gouvernement canadien de délaisser presque complètement la notion de négociation et d'affirmer à plusieurs reprises : « Aucune négociation en cas d'ambiguïté. » Les notions de procédure et de dialogue deviennent donc complètement dévaluées et le processus politique d'une sécession devient obscur et rigide.

La réponse québécoise ${ }^{22}$ n'est pas mieux, sinon pire. Le préambule s'ouvre avec 15 «CONSIDÉRANT que»; qui sont allégués comme étant des faits $^{23}$. Ces 15 faits aboutissent immédiatement à l'article 1, qui proclame que «Le peuple québécois peut, en fait et en droit, disposer de lui-même. Il est titulaire des droits universellement reconnus en vertu du principe de l'égalité de droits des peuples et de leur droit à disposer d'euxmêmes »; et puis, dans l'article 2: «Le peuple québécois a le droit inaliénable de choisir librement le régime politique et le statut juridique du Québec»; cela se

\footnotetext{
${ }^{21}$ Loi donnant effet à l'exigence de clarté formulée par la Cour suprême du Canada dans son avis sur le Renvoi relatif à la sécession du Québec, L.C., 2000, c. 26, C-31.8

${ }^{22}$ Loi sur l'exercice des droits fondamentaux et des prérogatives du peuple québécois et de l'État du Québec, L.R.Q., chapitre E-20.2. Nous soulignons.

${ }^{23}$ Mentionnons par ailleurs que si la plupart des considérants n'ont qu'une portée déclaratoire, certains relèvent davantage du jugement politique ; par exemple : «CONSIDÉRANT que le Québec fait face à une politique du gouvernement fédéral visant à remettre en cause la légitimité, l'intégrité et le bon fonctionnement de ses institutions démocratiques nationales » Cf. Yves-Marie MORISSETTE, Le renvoi sur la sécession du Québec. Bilan provisoire et perspectives, Montréal, Les éditions Varia, coll. Histoire et Société, 2001, page 23.
} 


\section{Åsbjørn Melkevik}

\section{La question de la sécession dans une fédération: Une critique des mauvaises interprétations}

complique encore avec l'article $3:$ :Le peuple québécois détermine seul, par l'entremise des institutions politiques qui lui appartiennent en propre, les modalités de l'exercice de son droit de choisir le régime politique et le statut juridique du Québec »; et encore avec l'article 4: «Lorsque le peuple québécois est consulté par un référendum tenu en vertu de la Loi sur la consultation populaire (chapitre C-64.1), l'option gagnante est celle qui obtient la majorité des votes déclarés valides, soit $50 \%$ de ces votes plus un vote. » C'est à se demander si les auteurs de cette loi ont compris quoi que ce soit au fédéralisme ou à la démocratie. Premièrement les faits ne justifient rien. Deuxièmement, du droit des peuples à disposer d'eux-mêmes nous ne pouvons pas faire découler ces «droits » qui sont proclamés, presque comme évidences. Troisièmement et principalement, la vision de la démocratie qui est présentée ici n'est absolument pas appropriée. Concernant la règle du $50 \%$ plus 1 vote, le professeur Jean-Pierre Derriennic a montré de manière assez convaincante la difficulté d'une telle règle dans les cas de sécession : « Nous acceptons la légitimité d'une décision prise par un vote que nous avons perdu, parce que nous savons qu'il y aura d'autres votes que parfois nous pourrons gagner. ${ }^{24}$ » Dans une sécession, il n'y a pas cette possibilité "d'autres votes » - qu'il suffise de dire qu'une sécession emportant 56\% des voix, même avec un fort taux de participation, serait soumise à de très fortes difficultés pour être effective. Mais là où nous voulons mettre l'accent, c'est que dans cette loi, la notion de délibération a totalement disparu : aucune négociation comme le souligne particulièrement l'article 3. Yves-Marie Morissette souligne que « la Loi no 99 tente d'assujettir entièrement et une fois pour toutes la norme constitutionnelle de clarté au seul contrôle de l'Assemblée nationale ${ }^{25}$ ». Le Québec, par l'entremise de son parlement, aurait donc un «droit» de déterminer seul, sans aucun dialogue ou consultation avec le reste du Canada, le régime politique et le statut juridique du Québec. L'application absurde de ce principe est de dire qu'il suffit de 50\% plus 1 des voix à un référendum pour que le Québec décide soit de devenir un régime républicain ou de se

\footnotetext{
${ }^{24}$ Jean-Pierre DERRIENNIC, Nationalisme et démocratie : réflexions sur les illusions des indépendantistes québécois, Montréal, Boréal, 1995, page 93. Ajoutons la célèbre citation de Rousseau : "plus les délibérations sont importantes et graves, plus l'avis qui l'emporte doit approcher de l'unanimité » $D u$ contrat social, Livre IV, ch. II.

${ }^{25}$ Yves-Marie MORISSETTE, op. cit., page 24.
} 


\section{Åsbjørn Melkevik La question de la sécession dans une fédération: Une critique des mauvaises interprétations}

séparer du Canada; évidemment nous exagérons, mais le principe de cette loi reste complètement inapproprié.

\section{La sécession comme projet négatif ou positif}

Dans la plupart des pays, il n’y a aucune procédure ou droit de sécession reconnu. La possibilité même de l'éventualité est rejetée catégoriquement par l'autorité centrale le Canada à cet égard est plutôt une exception. Ce rejet du droit de sécession est dans un certain sens positif, puisqu'il coupe court aux revendications farfelues; Hurst Hannum écrit : «In general, policymakers should continue to reject the notion that there is a legal right of secession. This principle will properly force nationalists to abandon their claim that "we are a nation and therefore have a right to a state." ${ }^{26}$ " Toutefois, l'absence de droit ou de procédure de sécession ne rend pas l'entreprise futile; toutes les revendications de sécession ne sont pas farfelues. La sécession devient alors beaucoup plus problématique, mais ne doit pas être considérée comme impossible. L'important est, comme nous allons le souligner, d'avoir un projet collectif - politique - qui rencontre un fort consensus dans la population et qui est mené à terme selon une procédure appropriée. Or, ici encore, des théories - comme celle de Stéphane Dion que nous allons examiner tentent de réduire la sécession à un simple calcul utilitariste entre les coûts et les bénéfices, ou entre la peur et la confiance. Cette réduction minimaliste de la sécession bien que parfois utile - est problématique sous de nombreux aspects. Seulement s'attacher à des éléments superficiels, mesurables, c'est oublier ou écarter le fait que la sécession n'est pas quelque chose de mesurable ou de prévisible par un calcul et que bien souvent les experts sont surpris par les événements; le politique est le domaine du possible et souvent de l'inconcevable. Nous examinerons ici premièrement une théorie négative de la sécession, pour deuxièmement proposer notre vision positive.

\footnotetext{
${ }^{26}$ Hurst HANNUM, op. cit., page 16.
} 


\section{Åsbjørn Melkevik}

\section{La question de la sécession dans une fédération: Une critique des mauvaises interprétations}

\section{La peur et la confiance}

Stéphane Dion défend, dans son article "Why is Secession Difficult in WellEstablished Democracies? Lessons from Quebec ${ }^{27} »$, que la possibilité d'une sécession est soumise à deux états émotifs : la peur et la confiance ${ }^{28}$. La logique en est que la peur entraînée par le gouvernement de l'union fédérale est essentielle pour une sécession autant que la confiance dans le projet sécessionniste: «They must fear the union and have confidence in secession. ${ }^{29}$ » Cela permet à Stéphane Dion de considérer la sécession comme un projet négatif et d'écarter, dans une certaine mesure, le côté positif du projet la sécession contre et non pour quelque chose. Une sécession serait selon Dion très improbable si l'État fédéral n'inspirait pas la crainte ${ }^{30}$. Encore une fois, l'application absurde de ce principe est de dire que le Québec n'a que très peu de chance d'être indépendant si le Canada ne menace pas directement la province, culturellement, économiquement ou politiquement. Cela est évidemment faux ; il y a plusieurs bonnes raisons qui peuvent mener à la sécession dans un État - fédéral ou non. L'examen des sécessions réussies - soit pacifique -, comme la Norvège et la Tchécoslovaquie, montre que si la peur joue un rôle, ce rôle est d'une importance dérisoire. Par contre, un examen des sécessions qui furent des échecs flagrants - sur le plan des moyens, donc dans lesquelles il y eut plusieurs morts, ce qui n'exclut pas qu'ils sont devenus de fait indépendant par la suite - pensons aux républiques fédérés yougoslaves, ou aux diverses tentatives de fragmentation en Afrique, l'examen montrerait qu'à ces endroits la peur fut bien un des facteurs qui entraîna la violence. Évidemment, il est impossible d'écarter la violence comme possibilité, si le Québec se séparait, mais de là à affirmer que la peur est en partie nécessaire à une sécession, c'est condamner toutes les sécessions à un risque de violence très élevé. La théorie de Dion n'est pas innocente ; introduire ce germe de peur,

\footnotetext{
${ }^{27}$ Stéphane DION, « Why is Secession Difficult in Well-Established Democracies? Lessons from Quebec ", British Journal of Political Science, vol. 26, no 2 (avril, 1996), p. 271.

${ }^{28}$ «Fear is defined here as the sense among members of a regional group that their cultural, economic or political situation will deteriorate within the existing union. Confidence is the sense among the group that it can perform better on its own and that secession is not too risky. »Ibid., page 271.

${ }^{29}$ Ibid., page 273.

${ }^{30}$ « Secessionist leaders have no chance if the union does not inspire fear among a significant proportion of the regional group's members. » Ibid., page 272.
} 


\section{Åsbjørn Melkevik La question de la sécession dans une fédération: Une critique des mauvaises interprétations}

et donc de possibilité de violence, dans la théorie même de la sécession lui permet de condamner le projet sécessionniste encore plus efficacement.

\section{L’ambivalence des projets sécessionnistes}

Selon nous, le nationalisme sécessionniste, pris dans son sens d'autodétermination, peut à la fois être très bien - s'il est fondé sur un réel projet politique avec un réel consensus de société - et très dangereux - s'il commence à être compris en termes de différence et de reconnaissance. Si le Québec aspire à être indépendant, souverain, son argumentation ne doit pas s'attacher à souligner que le Québec est une société distincte, mais doit bien plutôt expliquer le projet politique, les objectifs et la vision d'une société civique se reconnaissant dans un projet de souveraineté politique - l'argument ne doit donc pas être culturel ou historique, mais bien politique. La sortie du politique est sûrement le pire danger du nationalisme. Il faut selon l'expression de Hannum adopter une politique démocratique de «putting people first » : «Self-determination should be concerned primarily with people, not territory. ${ }^{31}$ " Stéphane Dion se trompe encore lorsqu'il affirme que "There has never been a single case of secession in democracies if we consider only the well-established ones, that is, those with at least 10 consecutive years of universal suffrage. ${ }^{32} »$ Cela est évidemment faux $^{33}$; la Norvège était bien une démocratie des plus sérieuses depuis 1898 lorsqu'elle a fait son indépendance de la Suède en 1905 - démocratie et sécession ne sont pas incompatibles.

L'important est, selon nous, de ne pas restreindre en théorie la possibilité d'une sécession en pratique. La sécession est réfractaire aux considérations «en théorie ». Ainsi, le propos de Hannum est insuffisant lorsqu'il écrit : "There are two instances in which secession should be supported by the international community. The first occurs

\footnotetext{
${ }^{31}$ Hurst HANNUM, op. cit., page 15.

${ }^{32}$ Stéphane DION, «The Dynamic of Secession: Scenarios after a Pro-Separatist Vote in a Quebec Referendum », Canadian Journal of Political Science, vol. 28, no 3 (Sep., 1995), pages 533-551

${ }^{33}$ La limite de 10 ans de suffrage universel est purement artificielle pour mesurer le sérieux d'une démocratie et nous ne la prenons donc pas en compte.
} 
when massive, discriminatory human rights violations, approaching the scale of genocide, are being perpetrated. [...] A second possible exception might find a right of secession if reasonable demands for local self-government or minority rights have been arbitrarily rejected by a central government - even without accompanying large-scale violence. ${ }^{34}$ » Nous sommes en accord avec ces deux cas de figure pour lesquels la communauté internationale devrait reconnaître la sécession, mais ces deux cas ne sont pas exclusifs; il y a d'autres circonstances acceptables pour une sécession. La sécession se situe quelque part entre la procédure et le projet; donc la sécession doit être faite de manière démocratique, pacifique et transparente d'une part et d'autre part reposer sur un réel projet de société. Évidemment que si l'immense majorité d'un État fédéré veut se séparer d'un État central et que cette volonté est confirmée par un référendum avec une question claire et qu'un sain dialogue s'établit entre les acteurs politiques pour discuter des modalités de la sécession, alors nous pourrons dire que la sécession doit être reconnue, en théorie et en pratique. Nous n'avons aucune bonne raison de croire qu'un tel scénario est impossible, sinon nos préjugés.

\section{Conclusion : une question non résolue}

Le type d'argumentation que nous avons essayé de développer tout au long de cet essai consiste à rejeter les dogmatismes ou les idéologies trop faciles. Charles de Gaulle disait : «Des chercheurs qui cherchent, on en trouve. Des chercheurs qui trouvent, on en cherche. » Or, justement, l'important est de chercher et d'être assez raisonnable pour ne pas affirmer avoir trouvé. Il ne faut pas fixer la question de la sécession - que l'on soit pour ou contre - ni par le Droit, ni par une quelconque politique rigide ou obscure; la sécession est une question politique et il faut par conséquent que ce soit le politique qui développe un projet collectif qui soit partagé par la population. Après, qu'il y ait une société distincte, une langue différente, un bagage historique commun ou non n'est pas l'élément déterminant en soi. Les différences sont souvent imaginées et créées artificiellement plus qu'autre chose : «Moins les différences sont importantes entre deux

${ }^{34}$ Hurst HANNUM, op. cit., page 16. 


\section{Åsbjørn Melkevik La question de la sécession dans une fédération: Une critique des mauvaises interprétations}

groupes, plus ils doivent lutter pour dépeindre ces différences comme absolues. ${ }^{35}$ » Disons donc au contraire de Ignatieff - qui affirme que « Le pouvoir est le vecteur qui transforme le mineur en majeur ${ }^{36} »-$ que le politique est précisément le vecteur qui permet de concilier les différences majeures en une harmonie sommes toutes fonctionnelle ; le fédéralisme en est très sûrement une expression. Or, si les différences, peu importe leur nature, sont telles qu'elles poussent à la désunion, alors «alea jacta est ».

${ }^{35}$ Michael IGNATIEFF, L'honneur du guerrier. Guerre ethnique et conscience moderne, Québec - Paris, Les Presses de l'Université Laval - Éditions La Découverte, coll. Prisme, 2000, page 50.

${ }^{36}$ Ibid., page 49. 


\section{Bibliographie}

DERRIENNIC, Jean-Pierre, Nationalisme et démocratie : réflexions sur les illusions des indépendantistes québécois, Montréal, Boréal, 1995, 144 pages.

DION, Stéphane, "Why is Secession Difficult in Well-Established Democracies? Lessons from Quebec », British Journal of Political Science, vol. 26, no. 2 (avril., 1996), pp. 269-283.

DION, Stéphane, «The Dynamic of Secession: Scenarios after a Pro-Separatist Vote in a Quebec Referendum », Canadian Journal of Political Science, vol. 28, no 3 (sep., 1995), pp. 533-551.

FRIEDRICH, Carl J., La démocratie constitutionnelle, Paris, Presses Universitaires de France, 1958, 564 pages.

FRIEDRICH, Carl J., Trends of Federalism in Theory and Practice, New York; Washington; Londres, Praeger Publishers, 1968, 193 pages.

HANNUM, Hurst, «The Specter of Secession: Responding to Claims for Ethnic SelfDetermination », Foreign Affairs, vol. 77, no 2, mars - avril, 1998, pp. 13-18.

IGNATIEFF, Michael, Blood and Belonging. Journeys Into The New Nationalism, Toronto, $\quad$ Penguin Canada, 1993, 326 pages.

IGNATIEFF, Michael, La Révolution des droits, Montréal, Boréal, 2001, 141 pages.

IGNATIEFF, Michael, L'honneur du guerrier. Guerre ethnique et conscience moderne, Québec - Paris, Les Presses de l'Université Laval - Éditions La Découverte, coll. Prisme, 2000, 210 pages.

Loi donnant effet à l'exigence de clarté formulée par la Cour suprême du Canada dans son avis sur le Renvoi relatif à la sécession du Québec, L.C., 2000, c. 26, C31.8 .

Loi sur l'exercice des droits fondamentaux et des prérogatives du peuple québécois et de l'État du Québec, L.R.Q., chapitre E-20.2.

MORISSETTE, Yves-Marie, Le renvoi sur la sécession du Québec. Bilan provisoire et perspectives, Montréal, Les éditions Varia, coll. Histoire et Société, 2001, 132 pages.

Renvoi relatif à la sécession du Québec, [1998] 2 R.C.S. 217. 

critique des mauvaises interprétations

WOOD, John R., "Secession: A Comparative Analytical Framework », Canadian Journal of Political Science, vol. 16, no 1, 1981, pp. 107-34. 